\title{
Some General Properties of Bi-shadowing Property
}

\author{
Mohammed Hussein Obaid Ajam Iftichar Mudhar Talb \\ Department of Mathematics, College of Education for Pure Science, \\ University of Babylon.Babylon,Iraq. \\ Email:pure.mohammed.hussein@uobabylon.edu.iq
}

\begin{abstract}
We concentrate on bi-shadowing property, it has important properties and applications in mathematics. In this paper some general properties of this concept are proved. Let $(Y, d)$ be a metric space $g, h:(Y, d) \rightarrow(Y, d)$ be maps have bi-shadowing property. We show the maps $g \circ h, g^{m}$ and $g \times h$ have bi-shadowing property.

Let $g, h:\left(\mathbb{R}^{n}, d\right) \rightarrow\left(\mathbb{R}^{n}, d\right)$ be maps on a metric space $\left(\mathbb{R}^{n}, d\right)$ have bi-shadowing property. We show the maps $g+h$ and $g . h$ have bi-shadowing property.

Key words: Pseudo-orbit, Shadowing property, Bi-Shadowing property.

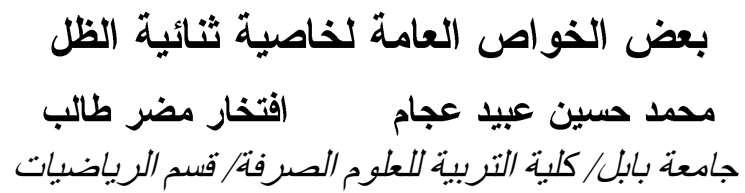

الخلاصة

حيث تم برهان انه لو كان للينا الدالتان h, و على الفضاء المتري (Y,d) كلاهما تمتلك خاصية ثنائية الظل فان

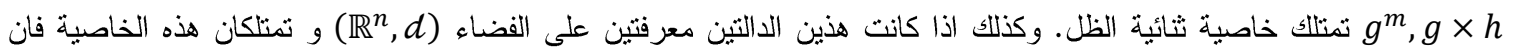

要

الكلمات المفتاحية: مسار كاذب، خاصية الظل، خاصية ثنائية الظل.
\end{abstract}

\section{1- Introduction}

Attractors of the discrete dynamical system on an infinite-time interval have been used to investigate the properties of the system. To do so, an arithmetic simulation of the attractors is needed, particularly for complicated system such as chaotic systems. Consequently, pseudo-orbits (approximated orbits) are now present, and the resultant behavior reflects the behavior of the approximated system only. Therefore, the question about the existence of a true orbit near a pseudo-orbit is obviously raised and strongly considered. It appears that the best way to carry out these ideas is by the concept of the property of (direct) shadowing. The concept of shadowing, in fact, plays a significant role in understanding the asymptotic behavior of dynamical systems; this goes back to 1960s, the work of [4].

The inverse idea is also important, that is, every true orbit of the system can be approximated by a pseudo orbit with specific properties. In practice, these pseudo orbits are taken from a pre-assigned class of orbits generated by continuous maps. This concept is called inverse shadowing, which was introduced by [8] and by [9] using $\delta$ method. A combination of the concepts shadowing and inverse shadowing is called bi-shadowing, which was introduced by [6], see also [5]. Bi-shadowing was considered in two cases, first case finite-dimensional systems in [6] and second case infinite dimensional systems in [3]. Bi-shadowing was also considered for set-valued dynamical systems in a metric space with an application to iterated function systems, see [1]. [7] studied the hyperbolic homeomorphisms on compact manifolds and presented 
both inverse shadowing and bi-shadowing properties with respect to a class of $\delta$ methods which are represented by continuous maps from the manifold into the space of bi-infinite sequences in the manifold with the product topology in [2] proved the concept of bi-shadowing with respect to continuous comparison maps for these systems under various conditions. Al-Badarneh also illustrated the finding by using examples of subclasses that incorporate: Kannan mappings, Chatterjea mappings and Reich mappings.

In this paper, some preliminaries needed are given, also we state and prove some general properties and theorems about bi-shadowing property.

\section{2- Preliminaries:}

Let $g:(Y, d) \rightarrow(Y, d)$ be a map defined on a metric space $(Y, d)$ and consider the dynamical system on $Y$ generated by the iterations of $g$, that is $g^{0}=i d_{y}$ and $\quad g^{n+1}=g^{n} \circ g$, for all $n \in \mathbb{N}$. We shall identify the map $g$ with the corresponding dynamical system. A sequence $\left\{y_{n}\right\}_{n=0}^{\infty} \subset Y$ is called a (true) orbit of $g$ if $\quad y_{n+1}=g\left(y_{n}\right)$, for all $n \in \mathbb{N}$. A sequence $\left\{z_{n}\right\}_{n=0}^{\infty} \subset Y$ is called a $\delta$ pseudo-orbit of $g$ if $d\left(z_{n+1}, g\left(z_{n}\right)\right) \leq \delta$ for all $n \in \mathbb{N}$ and for $\delta>0$.

Definition 2.1. [2]

A continuous map $g: Y \rightarrow Y$ is called bi-shadowing with respect to a comparison class of maps $C(Y)$ consisting of continuous maps on $Y$ and with positive parameters $a$ and $b$ if for any given $\delta$-pseudoorbit $\left\{x_{n}\right\}_{n=0}^{\infty}$ of $g$ with $0 \leq \delta \leq b$ and any $\varphi \in C(Y)$ satisfying: $\delta+\sup _{y \in Y} d(\varphi(y), g(y)) \leq b$ there exists a true orbit $\left\{y_{n}\right\}_{n=0}^{\infty}$ of $\varphi$ such that:

$$
d\left(x_{n}, y_{n}\right) \leq a\left(\delta+\sup _{y \in Y} d(\varphi(y), g(y))\right), \text { for all } n \in \mathbb{N} .
$$

\section{3- Main Theorems:}

In this section, we state and prove the main results about maps that have bi-shadowing property in a metric space $(Y, d)$ and $\left(Z, d^{\prime}\right)$.

Theorem 3.1.

If $g, h: Y \rightarrow Y$ are maps on $Y$ have the bi-shadowing property, then $g \circ h$ has the bi-shadowing property.

Proof:

Suppose that $g$ has bi-shadowing then for any given $\delta^{\prime}$-pseudo-orbit $\left\{y_{n}\right\}_{n=0}^{\infty}$ of $g$ with $0 \leq \delta^{\prime} \leq$ $b^{\prime}$ and any $\emptyset \in C(Y)$ satisfying :

$\delta^{\prime}+\sup _{y \in Y} d(\varnothing(y), g(y)) \leq b^{\prime}$

there exists a true orbit $\left\{\alpha_{n}\right\}_{n=0}^{\infty}$ of $\emptyset$ such that :

$d\left(y_{n}, \alpha_{n}\right) \leq a^{\prime}\left(\delta^{\prime}+\sup _{y \in Y} d(\varnothing(y), g(y))\right)$, for all $n \in \mathbb{N}$.

since $h$ has bi-shadowing then for any given $\delta^{\prime \prime}$-pseudo-orbit $\left\{z_{n}\right\}_{n=0}^{\infty}$ of $h$ with $0 \leq \delta^{\prime \prime} \leq b^{\prime \prime}$ and any $\theta \in C(Y)$ satisfying :

$\delta^{\prime \prime}+\sup _{y \in Y} d(\theta(y), h(y)) \leq b^{\prime \prime}$

there exists a true orbit $\left\{\beta_{n}\right\}_{n=0}^{\infty}$ of $\theta$ such that :

$d\left(z_{n}, \beta_{n}\right) \leq a^{\prime \prime}\left(\delta^{\prime \prime}+\sup _{y \in Y} d(\theta(y), h(y))\right)$, for all $n \in \mathbb{N}$.

Now, for some $r, r^{\prime} \geq 1$, let $a=r r^{\prime} a^{\prime \prime \prime} \quad$ when $a^{\prime \prime \prime}=\max \left\{a^{\prime}, a^{\prime \prime}\right\}, b=\min \left\{b^{\prime}, b^{\prime \prime}\right\}$, $b \geq \delta \geq \max \left\{\frac{\delta^{\prime}+\delta^{\prime \prime}}{r^{\prime}}, \frac{(r-1) b+\delta^{\prime}}{r}, \frac{(r-1) b+\delta^{\prime \prime}}{r}\right\} \geq 0$, for any given $\delta$-pseudo-orbit $\left\{x_{n}\right\}_{n=0}^{\infty}$ of $g \circ h$, and any $\varphi=\emptyset \circ \theta \in C(Y)$ satisfying :

$\delta+\sup _{y \in Y} d(\varphi(y), g \circ h(y)) \leq b$

$\sup _{y \in Y} d(\theta(\varnothing(y)), h(g(y))) \leq b-\delta$

$\sup _{y \in Y} d(\varnothing(y), g(y)) \leq r(b-\delta) \leq r b-r \frac{(r-1) b+\delta^{\prime}}{r} \leq b-\delta^{\prime} \leq b^{\prime}-\delta^{\prime}$

then there exists a true orbit $\left\{\alpha_{n}\right\}_{n=0}^{\infty}$ of $\emptyset$ such that : 


$$
d\left(y_{n}, \alpha_{n}\right) \leq a^{\prime}\left(\delta^{\prime}+\sup _{y \in Y} d(\varnothing(y), g(y))\right) \quad \text { for all } n \in \mathbb{N} .
$$

In the same way, we can get:

$$
\begin{aligned}
& \sup _{y \in Y} d(\theta(y), h(y)) \leq r(b-\delta) \leq r b-r \frac{(r-1) b+\delta^{\prime \prime}}{r} \leq b-\delta^{\prime \prime} \leq b^{\prime \prime}-\delta^{\prime \prime} \\
& \text { there exists a true orbit }\left\{\beta_{n}\right\}_{n=0}^{\infty} \text { of } \theta \text { such that : } \\
& d\left(z_{n}, \beta_{n}\right) \leq a^{\prime \prime}\left(\delta^{\prime \prime}+\sup _{y \in Y} d(\theta(y), h(y))\right) \quad \text { for all } n \in \mathbb{N} .
\end{aligned}
$$

Hence there exists a true orbit $\left\{x_{n}^{\prime}\right\}_{n=0}^{\infty}=\left\{\theta\left(\varnothing\left(x_{n}\right)\right)\right\}_{n=0}^{\infty}$ of $\varphi$ such that:

$$
\begin{aligned}
& \frac{1}{r} d\left(x_{n}, x_{n}^{\prime}\right) \leq d\left(y_{n}, \alpha_{n}\right)+d\left(z_{n}, \beta_{n}\right) \\
\leq & a^{\prime}\left(\delta^{\prime}+\sup _{y \in Y} d(\varnothing(y), g(y))\right)+a^{\prime \prime}\left(\delta^{\prime \prime}+\sup _{y \in Y} d(\theta(y), h(y))\right) \\
\leq & a^{\prime \prime \prime}\left(\delta^{\prime}+\sup _{y \in Y} d(\varnothing(y), g(y))\right)+a^{\prime \prime \prime}\left(\delta^{\prime \prime}+\sup _{y \in Y} d(\theta(y), h(y))\right) \\
& \leq a^{\prime \prime \prime}\left(\delta^{\prime}+\delta^{\prime \prime}+\sup _{y \in Y} d(\varnothing(y), g(y))+\sup _{y \in Y} d(\theta(y), h(y))\right) \\
& \leq a^{\prime \prime \prime}\left(\delta^{\prime}+\delta^{\prime \prime}+r^{\prime} \sup _{y \in Y} d(\theta(\varnothing(y)), h(g(y)))\right) \\
& \leq r^{\prime} a^{\prime \prime \prime}\left(\frac{\delta^{\prime}+\delta^{\prime \prime}}{r^{\prime}}+\sup _{y \in Y} d(\theta(\varnothing(y)), h(g(y)))\right) \\
\text { Hence } \quad & d\left(x_{n}, x_{n}^{\prime}\right) \leq r r^{\prime} a^{\prime \prime \prime}\left(\frac{\delta^{\prime}+\delta^{\prime \prime}}{r^{\prime}}+\sup _{y \in Y} d(\theta(\varnothing(y)), h(g(y)))\right) \\
& \leq a\left(\delta+\sup _{y \in Y} d(\theta(\varnothing(x)), h(g(x)))\right) \text { for all } n \in \mathbb{N} .
\end{aligned}
$$

Hence, $g \circ h$ has the bi-shadowing property.

\section{Corollary 3.2:}

Let $g: Y \rightarrow Y$ be a map. If $g$ has the bi-shadowing property, then $g^{k}$ has the bi-shadowing property for every $k \in \mathbb{N}$.

Proof:

We can prove this result by Induction Law of Theorem 3.1.

Let $\left(Y, d^{\prime}\right)$ and $\left(Z, d^{\prime \prime}\right)$ be metric space, $g: Y \rightarrow Y$ and $h: Z \rightarrow Z$ be maps ,the $d\left((y, z),\left(y^{\prime}, z^{\prime}\right)\right)=d^{\prime}\left(y, y^{\prime}\right)+d^{\prime \prime}\left(z, z^{\prime}\right)$.

We defined the map $(g \times h)(y, z)=(g(y), h(z))$, for all $y \in Y$ and for all $z \in Z$.

To prove that $(Y \times \mathrm{Z}, d)$ is a metric space.

Let $(y, z),\left(y^{\prime}, z^{\prime}\right)$ and $\left(y^{\prime \prime}, z^{\prime \prime}\right) \in Y \times \mathrm{Z}$.

1. Since $d\left((y, z),\left(y^{\prime}, z^{\prime}\right)\right)=d^{\prime}\left(y, y^{\prime}\right)+d^{\prime \prime}\left(z, z^{\prime}\right)$ also $d^{\prime}\left(y, y^{\prime}\right) \geq 0$ and $d^{\prime \prime}\left(z, z^{\prime}\right) \geq 0$. So that $d\left((y, z),\left(y^{\prime}, z^{\prime}\right)\right) \geq 0$.

2. $\quad d\left((y, z),\left(y^{\prime}, z^{\prime}\right)\right)=0$, if and only if $d^{\prime}\left(y, y^{\prime}\right)+d^{\prime \prime}\left(z, z^{\prime}\right)=0$,

if and only if $d^{\prime}\left(y, y^{\prime}\right)=0$ and $d^{\prime \prime}\left(z, z^{\prime}\right)=0$,

if and only if $y=y^{\prime}$ and $z=z^{\prime}$, thus $(y, z)=\left(y^{\prime}, z^{\prime}\right)$.

3. Since $d\left((y, z),\left(y^{\prime}, z\right)\right)=d^{\prime}\left(y, y^{\prime}\right)+d^{\prime \prime}\left(z, z^{\prime}\right)$

$=d^{\prime \prime}\left(z, z^{\prime}\right)+d^{\prime}\left(y, y^{\prime}\right)$

$=d\left(\left(y^{\prime}, z^{\prime}\right),(y, z)\right)$

Hence $d\left((y, z),\left(y^{\prime}, z^{\prime}\right)\right)=d\left(\left(y^{\prime}, z^{\prime}\right),(y, z)\right)$.

4. $\quad d\left((y, z),\left(y^{\prime}, z^{\prime}\right)\right)=d^{\prime}\left(y, y^{\prime}\right)+d^{\prime \prime}\left(z, z^{\prime}\right)$

$$
\begin{aligned}
& \leq d^{\prime}\left(y, y^{\prime \prime}\right)+d^{\prime \prime}\left(z, z^{\prime \prime}\right)+d^{\prime}\left(y^{\prime \prime}, y^{\prime}\right)+d^{\prime \prime}\left(z^{\prime \prime}, z^{\prime}\right) \\
& \leq\left[d^{\prime}\left(y^{\prime}, y^{\prime \prime}\right)+d^{\prime \prime}\left(z^{\prime}, z^{\prime \prime}\right)\right]+\left[d^{\prime}\left(y^{\prime \prime}, y^{\prime}\right)+d^{\prime \prime}\left(z^{\prime \prime}, z^{\prime}\right)\right]
\end{aligned}
$$




$$
\begin{gathered}
\leq d\left((y, z),\left(y^{\prime \prime}, z^{\prime \prime}\right)\right)+d\left(\left(y^{\prime \prime}, z^{\prime \prime}\right),\left(y^{\prime}, z^{\prime}\right)\right) \\
\text { Hence } d\left((y, z),\left(y^{\prime}, z^{\prime}\right)\right) \leq d\left((y, z),\left(y^{\prime \prime}, z^{\prime \prime}\right)\right)+d\left(\left(y^{\prime \prime}, z^{\prime \prime}\right),\left(y^{\prime}, z^{\prime}\right)\right)
\end{gathered}
$$

From $1,2,3$ and 4 , thus $(Y \times \mathrm{Z}, d)$ is a metric space.

Theorem 3.3:

Let $\left(Y, d^{\prime}\right)$ and $\left(Z, d^{\prime \prime}\right)$ be metric spaces, $g: Y \rightarrow Y$ and $h: Z \rightarrow Z$ be maps. if $g$ and $h$ has the bishadowing property then $g \times h$ has the bi-shadowing property.

Proof:

Let $\left(Y, d^{\prime}\right)$ and $\left(Z, d^{\prime \prime}\right)$ be metric spaces, $g: Y \rightarrow Y$ and $h: Z \rightarrow Z$ be maps, then we choose the metric $d$ on $Y \times Z$ as following:

For $x=\left(x_{1}, x_{2}\right), y=\left(y_{1}, y_{2}\right) \in Y \times Z, d(x, y)=d^{\prime}\left(x_{1}, y_{1}\right)+d^{\prime \prime}\left(x_{2}, y_{2}\right)$.

Suppose that $g$ has bi-shadowing then for any given $\delta^{\prime}$-pseudo-orbit $\left\{y_{n}\right\}_{n=0}^{\infty}$ of $g$ with $0 \leq \delta^{\prime} \leq b^{\prime}$ and any $\varnothing \in C(Y)$ satisfying: $\delta^{\prime}+\sup _{y \in Y} d^{\prime}(\varnothing(y), g(y)) \leq b^{\prime}$

there exists a true orbit $\left\{\alpha_{n}\right\}_{n=0}^{\infty}$ of $\emptyset$ such that

$d^{\prime}\left(y_{n}, \alpha_{n}\right) \leq a^{\prime}\left(\delta^{\prime}+\sup _{y \in Y} d^{\prime}(\varnothing(y), g(y))\right) \quad$ for all $n \in \mathbb{N}$.

Since $h$ has bi-shadowing then for any given $\delta^{\prime \prime}$-pseudo-orbit $\left\{z_{n}\right\}_{n=0}^{\infty}$ of $h$ with $0 \leq \delta^{\prime \prime} \leq b^{\prime \prime}$ and any $\theta \in C(Z)$ satisfying: $\delta^{\prime \prime}+\sup _{z \in Z} d^{\prime \prime}(\theta(z), h(z)) \leq b^{\prime \prime}$

there exists a true orbit $\left\{\beta_{n}\right\}_{n=0}^{\infty}$ of $\theta$ such that $d^{\prime \prime}\left(z_{n}, \beta_{n}\right) \leq a^{\prime \prime}\left(\delta^{\prime \prime}+\sup _{z \in Z} d^{\prime \prime}(\theta(z), h(z))\right) \quad$ for all $n \in \mathbb{N}$.

Now, let $a=\max \left\{a^{\prime}, a^{\prime \prime}\right\}, \quad b=\min \left\{b^{\prime}, b^{\prime \prime}\right\}, \quad b \geq \delta \geq \delta^{\prime}+\delta^{\prime \prime} \geq 0$, for any given $\delta$-pseudo-orbit $\left\{x_{n}\right\}_{n=0}^{\infty}=\left\{\left(y_{n}, z_{n}\right)\right\}_{n=0}^{\infty}$ of $g \times h$ and any $\varphi \in C(Y \times Z)$ satisfying:

$\delta+\sup _{(y, z) \in Y \times Z} d(\varphi(y, z),(g \times h)(y, z)) \leq b$

$\sup _{(y, z) \in Y \times z} d(\varphi(y, z),(g \times h)(y, z)) \leq b-\delta$

since $\varphi(y, z)=(\varnothing(y), \theta(z))$

$\sup _{y \in Y} d^{\prime}(\varnothing(y), g(y))+\sup _{z \in Z} d^{\prime \prime}(\theta(z), h(z)) \leq b-\delta$

Hence $\sup _{y \in Y} d^{\prime}(\varnothing(y), g(y)) \leq b-\delta \leq b^{\prime}-\delta^{\prime}$

Then there exists a true orbit $\left\{\alpha_{n}\right\}_{n=0}^{\infty}$ of $\emptyset$ such that

$$
d^{\prime}\left(y_{n}, \alpha_{n}\right) \leq a^{\prime}\left(\delta^{\prime}+\sup _{y \in Y} d^{\prime}(\varnothing(y), g(y))\right) \quad \text { for all } n \in \mathbb{N} .
$$

And also $\sup _{z \in Z} d^{\prime \prime}(\theta(z), h(z)) \leq b-\delta \leq b^{\prime \prime}-\delta^{\prime \prime}$

Then there exists a true orbit $\left\{\beta_{n}\right\}_{n=0}^{\infty}$ of $\theta$ such that

$$
d^{\prime \prime}\left(z_{n}, \beta_{n}\right) \leq a^{\prime \prime}\left(\delta^{\prime \prime}+\sup _{z \in Z} d^{\prime \prime}(\theta(z), h(z))\right) \quad \text { for all } n \in \mathbb{N} .
$$

Hence there exists a true orbit $\left\{x_{n}^{\prime}\right\}_{n=0}^{\infty}=\left\{\left(\alpha_{n}, \beta_{n}\right)\right\}_{n=0}^{\infty}$ of $\varphi$ such that

$$
\begin{aligned}
d\left(x_{n}, x_{n}^{\prime}\right) \leq d & \left(\left(y_{n}, z_{n}\right),\left(\alpha_{n}, \beta_{n}\right)\right) \leq d^{\prime}\left(y_{n}, \alpha_{n}\right)+d^{\prime \prime}\left(z_{n}, \beta_{n}\right) \\
& \leq a^{\prime}\left(\delta^{\prime}+\sup _{y \in Y} d^{\prime}(\varnothing(y), g(y))\right)+a^{\prime \prime}\left(\delta^{\prime \prime}+\sup _{z \in Z} d^{\prime \prime}(\theta(z), h(z))\right) \\
& \leq a\left(\delta^{\prime}+\sup _{y \in Y} d^{\prime}(\varnothing(y), g(y))\right)+a\left(\delta^{\prime \prime}+\sup _{z \in Z} d^{\prime \prime}(\theta(z), h(z))\right) \\
& \leq a\left(\delta^{\prime}+\delta^{\prime \prime}+\sup _{y \in Y} d^{\prime}(\varnothing(y), g(y))+\sup _{z \in Z} d^{\prime \prime}(\theta(z), h(z))\right) \\
& \leq a\left(\delta+\sup _{(y, z) \in Y \times Z} d(\varphi(y, z),(g \times h)(y, z))\right)
\end{aligned}
$$

Hence $g \times h$ has the bi-shadowing property.

Now, we state and prove the main results about maps have bi-shadowing property in a metric $\operatorname{space}\left(\mathbb{R}^{n}, d\right)$.

Theorem 3.4: 
Let $g, h:\left(\mathbb{R}^{n}, d\right) \rightarrow\left(\mathbb{R}^{n}, d\right)$ be maps. if $g$ and $h$ has the bi-shadowing property then $g+h$ has the bi-shadowing property .

Proof: Suppose that $g$ has bi-shadowing then for any given $\delta^{\prime}$-pseudo-orbit $\left\{y_{n}\right\}_{n=0}^{\infty}$ of $g$ with $0 \leq \delta^{\prime} \leq b^{\prime}$ and any $\emptyset \in C\left(\mathbb{R}^{n}\right)$ satisfying :

$\delta^{\prime}+\sup _{y \in \mathbb{R}^{n}} d(\varnothing(y), g(y)) \leq b^{\prime}$

there exists a true orbit $\left\{\alpha_{n}\right\}_{n=0}^{\infty}$ of $\emptyset$ such that

$d\left(y_{n}, \alpha_{n}\right) \leq a^{\prime}\left(\delta^{\prime}+\sup _{y \in \mathbb{R}^{n}} d(\varnothing(y), g(y))\right), \quad$ for all $n \in \mathbb{N}$.

And $h$ has bi-shadowing then for any given $\delta^{\prime \prime}$-pseudo-orbit $\left\{z_{n}\right\}_{n=0}^{\infty}$ of $h$ with $0 \leq \delta^{\prime \prime} \leq b^{\prime \prime}$ and any $\theta \in C\left(\mathbb{R}^{n}\right)$ satisfying:

$$
\delta^{\prime \prime}+\sup _{y \in \mathbb{R}^{n}} d(\theta(y), h(y)) \leq b^{\prime \prime}
$$

there exists a true orbit $\left\{\beta_{n}\right\}_{n=0}^{\infty}$ of $\theta$ such that

$$
d\left(z_{n}, \beta_{n}\right) \leq a^{\prime \prime}\left(\delta^{\prime \prime}+\sup _{y \in \mathbb{R}^{n}} d(\theta(y), h(y))\right), \quad \text { for all } n \in \mathbb{N} .
$$

Now, for some $r, r^{\prime} \geq 1$, let $a=r^{\prime} a^{\prime \prime \prime} \quad$ when $a^{\prime \prime \prime}=\max \left\{a^{\prime}, a^{\prime \prime}\right\}, b=\min \left\{b^{\prime}, b^{\prime \prime}\right\}$, $b \geq \delta \geq \max \left\{\frac{\delta^{\prime}+\delta^{\prime \prime}}{r^{\prime}}, \frac{(r-1) b+\delta^{\prime}}{r}, \frac{(r-1) b+\delta^{\prime \prime}}{r}\right\} \geq 0$, for any given $\delta$-pseudo-orbit $\quad\left\{x_{n}\right\}_{n=0}^{\infty}=\left\{\left(y_{n}+z_{n}\right)\right\}_{n=0}^{\infty}$ of $g+h$, let $\varphi \in C\left(\mathbb{R}^{n}\right)$ when $\quad \varphi(y)=(\varnothing(y)+\theta(y))$ for all $y \in \mathbb{R}^{n}$ satisfying:

$$
\begin{aligned}
& \delta+\sup _{y \in \mathbb{R}^{n}} d(\varphi(y),(g+h)(y)) \leq b \\
& \sup _{y \in \mathbb{R}^{n} d((\varnothing(y)+\theta(y)),(g(y)+h(y))) \leq b-\delta} \sup _{y \in \mathbb{R}^{n}} d(\varnothing(y), g(y))+\sup _{y \in \mathbb{R}^{n}} d(\theta(y), h(y)) \leq r(b-\delta)
\end{aligned}
$$

Hence $\sup _{y \in \mathbb{R}^{n}} d(\varnothing(y), g(y)) \leq r(b-\delta)$

$$
\sup _{y \in \mathbb{R}^{n}} d(\varnothing(y), g(y)) \leq r b-r \delta \leq r b-r \frac{(r-1) b+\delta^{\prime}}{r}=b-\delta^{\prime} \leq b^{\prime}-\delta^{\prime}
$$

there exists a true orbit $\left\{\alpha_{n}\right\}_{n=0}^{\infty}$ of $\emptyset$ such that And also: $d\left(y_{n}, \alpha_{n}\right) \leq a^{\prime}\left(\delta^{\prime}+\sup _{y \in \mathbb{R}^{n}} d(\varnothing(y), g(y))\right), \quad$ for all $n \in \mathbb{N}$

$$
\begin{aligned}
& \sup _{y \in \mathbb{R}^{n}} d(\theta(y), h(y)) \leq r(b-\delta) \\
& \sup _{y \in \mathbb{R}^{n}} d(\theta(y), h(y)) \leq r b-r \delta \leq r b-r \frac{(r-1) b+\delta^{\prime \prime}}{r}=b-\delta^{\prime \prime} \leq b^{\prime \prime}-\delta^{\prime \prime}
\end{aligned}
$$

there exists a true orbit $\left\{\beta_{n}\right\}_{n=0}^{\infty}$ of $\theta$ such that $d\left(z_{n}, \beta_{n}\right) \leq a^{\prime \prime}\left(\delta^{\prime \prime}+\sup _{y \in \mathbb{R}^{n}} d(\theta(y), h(y))\right), \quad$ for all $n \in \mathbb{N}$

Hence there exists a true orbit $\left\{x_{n}^{\prime}\right\}_{n=0}^{\infty}=\left\{\alpha_{n}+\beta_{n}\right\}_{n=0}^{\infty}$ of $\varphi$ such that

$$
\begin{gathered}
d\left(x_{n}, x_{n}^{\prime}\right) \leq d\left(\left(y_{n}+z_{n}\right),\left(\alpha_{n}+\beta_{n}\right)\right) \leq d\left(y_{n}, \alpha_{n}\right)+d\left(z_{n}, \beta_{n}\right) \\
\leq a^{\prime}\left(\delta^{\prime}+\sup _{y \in \mathbb{R}^{n}} d(\varnothing(y), g(y))\right)+a^{\prime \prime}\left(\delta^{\prime \prime}+\sup _{y \in \mathbb{R}^{n}} d(\theta(y), h(y))\right) \\
\leq a^{\prime \prime \prime}\left(\delta^{\prime}+\sup _{y \in \mathbb{R}^{n}} d(\emptyset(y), g(y))\right)+a^{\prime \prime \prime}\left(\delta^{\prime \prime}+\sup _{y \in \mathbb{R}^{n}} d(\theta(y), h(y))\right) \\
\leq a^{\prime \prime \prime}\left(\delta^{\prime}+\delta^{\prime \prime}+\sup _{y \in \mathbb{R}^{n}} d(\emptyset(y), g(y))+\sup _{y \in \mathbb{R}^{n}} d(\theta(y), h(y))\right) \\
\leq a^{\prime \prime \prime}\left(\delta^{\prime}+\delta^{\prime \prime}+r^{\prime} \sup _{y \in \mathbb{R}^{n}} d(\varphi(y),(g(y)+h(y)))\right) \\
\leq r^{\prime} a^{\prime \prime \prime}\left(\frac{\delta^{\prime}+\delta^{\prime \prime}}{r^{\prime}}+\sup _{y \in \mathbb{R}^{n}} d(\varphi(y),(g(y)+h(y)))\right) \\
\text { Hence } d\left(x_{n}, x_{n}^{\prime}\right) \leq r^{\prime} a^{\prime \prime \prime}\left(\frac{\delta^{\prime}+\delta^{\prime \prime}}{r^{\prime}}+\sup _{y \in \mathbb{R}^{n}} d(\varphi(y),(g(y)+h(y)))\right) \\
\leq a\left(\delta+\sup _{y \in \mathbb{R}^{n}} d(\varphi(y),(g(y)+h(y)))\right)
\end{gathered}
$$

Hence $g+h$ has the bi-shadowing property.

Theorem 3.5: 
Let $g, h:\left(\mathbb{R}^{n}, d\right) \rightarrow\left(\mathbb{R}^{n}, d\right)$ be maps. if $g$ and $h$ has the bi-shadowing property then $g$. $h$ has the bi-shadowing property .

Proof: Suppose that $g$ has bi-shadowing then for any given $\delta^{\prime}$-pseudo-orbit $\left\{y_{n}\right\}_{n=0}^{\infty}$ of $g$ with $0 \leq \delta^{\prime} \leq b^{\prime}$ and any $\emptyset \in C\left(\mathbb{R}^{n}\right)$ satisfying :

$\delta^{\prime}+\sup _{y \in \mathbb{R}^{n}} d(\varnothing(y), g(y)) \leq b^{\prime}$

there exists a true orbit $\left\{\alpha_{n}\right\}_{n=0}^{\infty}$ of $\emptyset$ such that

$d\left(y_{n}, \alpha_{n}\right) \leq a^{\prime}\left(\delta^{\prime}+\sup _{y \in \mathbb{R}^{n}} d(\varnothing(y), g(y))\right), \quad$ for all $n \in \mathbb{N}$.

And $h$ has bi-shadowing then for any given $\delta^{\prime \prime}$-pseudo-orbit $\left\{z_{n}\right\}_{n=0}^{\infty}$ of $h$ with $0 \leq \delta^{\prime \prime} \leq b^{\prime \prime}$ and any $\theta \in C\left(\mathbb{R}^{n}\right)$ satisfying :

$$
\delta^{\prime \prime}+\sup _{y \in \mathbb{R}^{n}} d(\theta(y), h(y)) \leq b^{\prime \prime}
$$

there exists a true orbit $\left\{\beta_{n}\right\}_{n=0}^{\infty}$ of $\theta$ such that

$$
d\left(z_{n}, \beta_{n}\right) \leq a^{\prime \prime}\left(\delta^{\prime \prime}+\sup _{y \in \mathbb{R}^{n}} d(\theta(y), h(y))\right), \quad \text { for all } n \in \mathbb{N} .
$$

Now, for some $r, r^{\prime} \geq 1$, let $a=r r^{\prime} a^{\prime \prime \prime} \quad$ when $a^{\prime \prime \prime}=\max \left\{a^{\prime}, a^{\prime \prime}\right\}, b=\min \left\{b^{\prime}, b^{\prime \prime}\right\}$, $b \geq \delta \geq \max \left\{\frac{\delta^{\prime}+\delta^{\prime \prime}}{r^{\prime}}, \frac{(r-1) b+\delta^{\prime}}{r}, \frac{(r-1) b+\delta^{\prime \prime}}{r}\right\} \geq 0$, for any given $\delta$-pseudo-orbit $\quad\left\{x_{n}\right\}_{n=0}^{\infty}=\left\{\left(y_{n} \cdot z_{n}\right)\right\}_{n=0}^{\infty}$ of $\quad g . h$, let $\varphi \in C\left(\mathbb{R}^{n}\right) \quad$ when $\quad \varphi(y)=(\varnothing(y) \cdot \theta(y)) \quad$ for $\quad$ all $\quad y \in \mathbb{R}^{n}$ satisfying: $\delta+\sup _{y \in \mathbb{R}^{n}} d(\varphi(y),(g \cdot h)(y)) \leq b$

$$
\begin{aligned}
& \sup _{y \in \mathbb{R}^{n}} d((\varnothing(y) \cdot \theta(y)),(g(y) \cdot h(y))) \leq b-\delta \\
& \sup _{y \in \mathbb{R}^{n}} d(\varnothing(y), g(y))+\sup _{y \in \mathbb{R}^{n}} d(\theta(y), h(y)) \leq r(b-\delta)
\end{aligned}
$$

Hence $\sup _{y \in \mathbb{R}^{n}} d(\varnothing(y), g(y)) \leq r(b-\delta)$

$$
\sup _{y \in \mathbb{R}^{n}} d(\varnothing(y), g(y)) \leq r b-r \delta \leq r b-r \frac{(r-1) b+\delta^{\prime}}{r}=b-\delta^{\prime} \leq b^{\prime}-\delta^{\prime}
$$

there exists a true orbit $\left\{\alpha_{n}\right\}_{n=0}^{\infty}$ of $\emptyset$ such that $d\left(y_{n}, \alpha_{n}\right) \leq a^{\prime}\left(\delta^{\prime}+\sup _{y \in \mathbb{R}^{n}} d(\varnothing(y), g(y))\right), \quad$ for all $n \in \mathbb{N}$

And also: $\quad \sup _{y \in \mathbb{R}^{n}} d(\theta(y), h(y)) \leq r(b-\delta)$

$$
\begin{aligned}
& \sup _{y \in \mathbb{R}^{n}} d(\theta(y), h(y)) \leq r b-r \delta \leq r b-r \frac{(r-1) b+\delta^{\prime \prime}}{r}=b-\delta^{\prime \prime} \leq b^{\prime \prime}-\delta^{\prime \prime} \\
& \text { there exists a true orbit }\left\{\beta_{n}\right\}_{n=0}^{\infty} \text { of } \theta \text { such that } \\
& d\left(z_{n}, \beta_{n}\right) \leq a^{\prime \prime}\left(\delta^{\prime \prime}+\sup _{y \in \mathbb{R}^{n}} d(\theta(y), h(y))\right), \quad \text { for all } n \in \mathbb{N}
\end{aligned}
$$

Hence there exists a true orbit $\left\{x_{n}^{\prime}\right\}_{n=0}^{\infty}=\left\{\alpha_{n} \cdot \beta_{n}\right\}_{n=0}^{\infty}$ of $\varphi$ such that

$$
\begin{aligned}
& \frac{1}{r} d\left(x_{n}, x_{n}^{\prime}\right) \leq \frac{1}{r} d\left(\left(y_{n}, z_{n}\right),\left(\alpha_{n} \cdot \beta_{n}\right)\right) \leq d\left(y_{n}, \alpha_{n}\right)+d\left(z_{n}, \beta_{n}\right) \\
& \leq a^{\prime}\left(\delta^{\prime}+\sup _{y \in \mathbb{R}^{n}} d(\varnothing(y), g(y))\right)+a^{\prime \prime}\left(\delta^{\prime \prime}+\sup _{y \in \mathbb{R}^{n}} d(\theta(y), h(y))\right) \\
& \leq a^{\prime \prime \prime}\left(\delta^{\prime}+\sup _{y \in \mathbb{R}^{n}} d(\varnothing(y), g(y))\right)+a^{\prime \prime \prime}\left(\delta^{\prime \prime}+\sup _{y \in \mathbb{R}^{n}} d(\theta(y), h(y))\right) \\
& \leq a^{\prime \prime \prime}\left(\delta^{\prime}+\delta^{\prime \prime}+\sup _{y \in \mathbb{R}^{n}} d(\varnothing(y), g(y))+\sup _{y \in \mathbb{R}^{n}} d(\theta(y), h(y))\right) \\
& \leq a^{\prime \prime \prime}\left(\delta^{\prime}+\delta^{\prime \prime}+r^{\prime} \sup _{y \in \mathbb{R}^{n}} d(\varphi(y),(g(y) \cdot h(y)))\right) \\
& \leq r^{\prime} a^{\prime \prime \prime}\left(\frac{\delta^{\prime}+\delta^{\prime \prime}}{r^{\prime}}+\sup _{y \in \mathbb{R}^{n}} d(\varphi(y),(g(y) \cdot h(y)))\right) \\
& \text { Hence } d\left(x_{n}, x_{n}^{\prime}\right) \leq r r^{\prime} a^{\prime \prime \prime}\left(\frac{\delta^{\prime}+\delta^{\prime \prime}}{r}+\sup _{y \in \mathbb{R}^{n}} d(\varphi(y),(g(y) \cdot h(y)))\right) \\
& \leq a\left(\delta+\sup _{y \in \mathbb{R}^{n}} d(\varphi(y),(g(y) \cdot h(y)))\right)
\end{aligned}
$$

Hence $g . h$ has the bi-shadowing property. 


\section{References}

[1] Al-Badarneh, A. Bi-shadowing of Contractive Set-Valued Mappings with Application to IFS's: The Non-Convex Case. JJMS, 2014, 7(4), pp. 287-301.

[2] Al-Badarneh, A. Bi-Shadowing of Some Classes of Single-Valued Almost Contractions. Applied Mathematical Sciences, 2015, 9(58), pp. 2859-2869.

[3] Anosov, D. Geodesic flows and closed Riemannian manifolds with negative curvature. Proc. Steklov Inst. Math. 90, 1967.

[4] Bowen, R. Equilibrium States and the Ergodic Theory of Anosov Diffeomorphisms. Springer-Verlag, 1970.

[5] Diamond, P., Kloeden, P., Kozyakin, V., and Pokrovskii, A. Computer Robustness of semi-hyperbolic mappings. Random and computational Dynamics, 3, 1995, pp. 53-70.

[6] Diamond, P., Kloeden, P., Kozyakin, V., and Pokrovskii, A. Semi-Hyperbolicity and Bi-Shadowing. American Institute of Mathematical Sciences, 2012.

[7] Kloeden, P., and Ombach, J. Australian Resarch Council Grant. Hyperbolic homeomorphisms and bishadowing, LXV.2, 1997, pp. 171-177.

[8] Kloeden, P., Ombach, J., and Pokrovskii, A. Continuous and inverse shadowing. J. Funct. Differ. Equ., 6, 1999, pp. 135-151.

[9] Pilyugin, S. Shadowing in Dynamical Systems. Lecture Notes in Mathematics, 1706,Springer-Verlag, Berlin, 1999. 